\title{
The Role of Pediatric BCG Vaccine in Type 1 Diabetes Onset
}

\author{
John Doupis - Konstantinos Kolokathis - Eftychia Markopoulou • \\ Vasiliki Efthymiou - George Festas · Vasiliki Papandreopoulou • \\ Chrysoula Kallinikou · Despina Antikidou • Golfo Gemistou • \\ Theodoros Angelopoulos
}

Received: August 3, 2021 / Accepted: September 22, 2021 / Published online: October 1, 2021

(C) The Author(s) 2021

\begin{abstract}
Introduction: Bacille Calmette-Guérin (BCG) vaccination has shown promising therapeutic effects for type 1 diabetes (T1D). According to recent studies, immunometabolism modification and regulation of $\mathrm{T}$ lymphocytes constitute the proposed mechanisms by which BCG vaccination may delay T1D onset. Clinical trial evidence from Turkey supports that two to three doses of the BCG vaccine in childhood, with the first dose administered in the first year of life, may prevent T1D. In the same study, one or zero vaccinations appeared to have no effect in
\end{abstract}

J. Doupis $(\bowtie) \cdot$ E. Markopoulou · G. Festas Internal Medicine and Diabetes Department, Salamis Naval and Veterans Hospital, Salamis, Greece

e-mail: john.doupis@harvard-alumni.org

J. Doupis - K. Kolokathis - V. Papandreopoulou · C. Kallinikou · D. Antikidou · T. Angelopoulos Iatriko Paleou Falirou Medical Center, Diabetes Clinic, Athens, Greece

\section{Efthymiou}

University Research Institute of Maternal and Child Health and Precision Medicine, and UNESCO Chair on Adolescent Health Care, National and

Kapodistrian University of Athens Medical School, 'Aghia Sophia' Children's Hospital, 11527 Athens, Greece

G. Gemistou

Pan-Hellenic Federation of People with Diabetes, Athens, Greece
T1D onset prevention. In Greece, the BCG vaccine was administered in a single dose at the age of 9 years in elementary school. BCG vaccination was not performed on a mandatory basis, creating one BCG vaccinated and one nonvaccinated population. The aim of our study was to investigate the possible effect of a single dose of BCG vaccine, at the age of 9 years, on the time of T1D onset, in a population of BCG vaccinated and non-vaccinated patients with diagnosed T1D.

Methods: To test this hypothesis, a survey through the Pan-Hellenic Federation of People with Diabetes (PFPD) was performed. In this observational, retrospective study, participating patients provided information regarding age, gender, time of diagnosis, and BCG vaccination status. Patients diagnosed with T1D before the age of 9 years were excluded from the analysis. Results: The final sample included 196 patients (73 male and 123 female) with a mean age of $42.2 \pm 14.3$ years and a mean duration of diabetes of $16.8 \pm 12.9$ years. Mean age of T1D diagnosis in the BCG vaccinated group was $24.0 \pm 19.0$ years, while the mean age of T1D diagnosis in the BCG non-vaccinated group was $21.5 \pm 14.3$ years $(p=0.03)$. No interaction was found between gender and the age of diagnosis for BCG vaccinated and unvaccinated patients $(p=0.86)$.

Conclusion: The results of our study suggest that a single dose of BCG vaccine, performed at the age of 9 years, may delay the onset of T1D 
by 2.5 years. Additional studies of children receiving multiple doses of BCG should be conducted to possibly prove prolongation of the disease-free interval.

Keywords: Diabetes; Type 1 diabetes; BCG vaccine; Immunometabolism; Hygiene hypothesis

\section{Key Summary Points}

Why carry out this study?

Approximately 463 million people are living with diabetes worldwide.

Clinical trials support that bacille Calmette-Guérin (BCG) vaccination possibly delays type 1 diabetes (T1D) onset via modification of immunometabolism and inflammation.

In Greece BCG vaccination was not performed on a mandatory basis, creating one BCG vaccinated and one nonvaccinated population.

\section{What was learned from the study?}

Differences between the age of diagnosis and BCG vaccination status group were tested by the Mann-Whitney $U$ test.

The difference between the two groups was statistically significant $(p=0.03)$. The BCG non-vaccination group of patients tends to have the initial diagnosis earlier than vaccinated patients.

The current trial showed that BCG vaccination, with a single dose at the age of 9 years, probably causes a delay in T1D onset.

\section{INTRODUCTION}

The global estimate of adults living with diabetes is 463 million, while a further 1.1 million children and adolescents living with type 1 diabetes (T1D) [1]. Two decades of research have indicated the beneficial effect of bacille Calmette-Guérin (BCG) vaccination for T1D. BCG is one of the commonest vaccines worldwide, and it is has been used for over 100 years with limited side effects. Clinical trials support that BCG vaccination can delay or even prevent the onset of T1D via modification of the immune system response and immunometabolism [2].

According to the immunometabolism mechanism, after BCG vaccination, immune system cells shift their metabolic function from overactive oxidative phosphorylation to aerobic glycolysis. Oxidative phosphorylation depends on small amounts of glucose to produce energy via the Krebs cycle. On the contrary, aerobic glycolysis demands great amounts of extracellular glucose to obtain energy and synthesize purines, which are DNA and RNA building blocks [2]. In addition, BCG vaccination enhances tumor necrosis factor (TNF) production, which activates $\mathrm{T}$ regulatory cells (Tregs) and eliminates T cytotoxic lymphocytes (CTL). As a result, the autoimmune response of CTLs against insulin-produced islet of the pancreas is reduced due to the CTLs limited number and the suppressive action of Tregs [3-5].

Clinical trial evidence from Turkey supports that two to three doses of the BCG vaccine in childhood, with the first dose administered in the first year of life, may prevent T1D [6]. In Greece, the BCG vaccine was administered in a single dose at the age of 9 years in elementary school as a part of the national vaccination program. BCG vaccination was not performed on a mandatory basis, creating one BCG vaccinated and one non-vaccinated population. The aim of our study was to investigate the possible effect of a single dose of BCG vaccine, at the age of 9 years, on the time of T1D onset, in a population of BCG vaccinated and non-vaccinated patients with diagnosed T1D.

\section{METHODS}

To test this hypothesis, a retrospective observational study, through the Pan-Hellenic Federation of People with Diabetes (PFPD), was performed. Participating patients with 


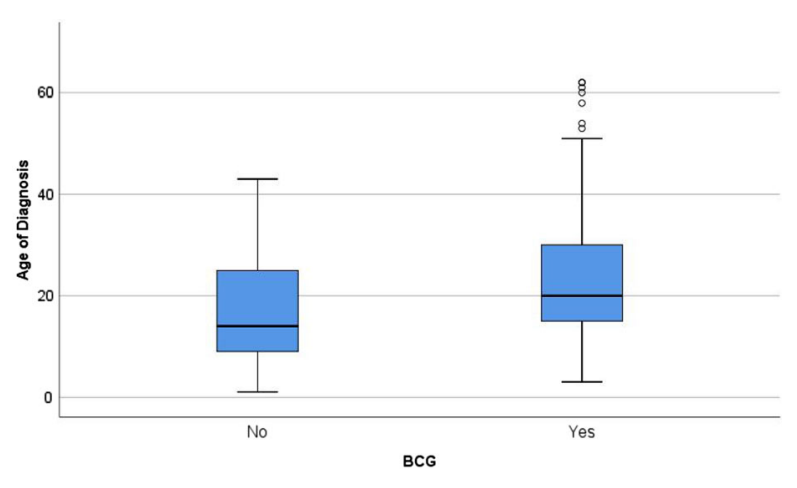

Fig. 1 Boxplot of age of diagnosis in bacille Calmette-Guérin (BCG) vaccination groups

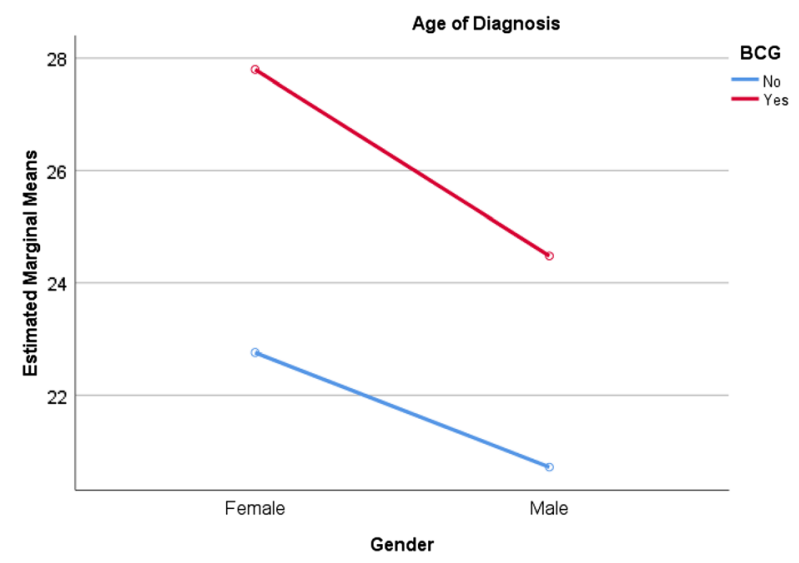

Fig. 2 Line chart of interaction between gender and age of diagnosis for bacille Calmette-Guérin (BCG) vaccination groups

diagnosed T1D provided information regarding age, gender, time of diagnosis, and BCG vaccination status. Patients diagnosed with T1D before the age of 9 years were excluded from the analysis. The study was conducted from January 2019 to June 2019.

Differences between the age of diagnosis and BCG vaccination status group were tested by the Mann-Whitney $U$ test. Two-way analysis of variance (ANOVA) after logarithmic transformation of the data was used to explore the interaction with gender. Statistical analysis was conducted with SPSS software version 25 for Windows (IBM Corp. Released 2017. IBM SPSS Statistics for Windows, Version 25.0. Armonk, NY: IBM Corp.).
The study protocol was conducted according to the Declaration of Helsinki and approved by the ethics committee of the Salamis Naval and Veterans Hospital, Salamis, Greece.

\section{RESULTS}

The final sample included 196 patients diagnosed with T1D (73 male and 123 female) with a mean age ( \pm standard deviation) of $42.2 \pm 14.3$ years and a mean ( \pm standard deviation) duration of diabetes $16.8 \pm 12.9$ years.

The median ( \pm interquartile range) age of diagnosis in the BCG vaccinated group of patients was $24.0 \pm 19.0$ years, while the median ( \pm interquartile range) age of the BCG nonvaccinated group of patients was $21.5 \pm 14.3$ years $(p=0.03)$ (Fig. 1$)$. The difference between the two groups was statistically significant. The BCG non-vaccinated group of patients tended to have the initial diagnosis earlier than vaccinated patients.

No interaction was found between gender and the age of diagnosis for BCG vaccinated and non-vaccinated patients $(p=0.86)$ (Fig. 2). For male patients, the median ( \pm interquartile range) age of diagnosis in the BCG vaccinated group was $25.0 \pm 15.5$ years, while the median ( \pm interquartile range) age of the BCG nonvaccinated group was $20.5 \pm 14.5$ years. For female patients, the median ( \pm interquartile range) age of diagnosis in the BCG vaccinated group was $24.0 \pm 21.3$ years, while the median ( \pm interquartile range) age of the BCG nonvaccinated group was $21.0 \pm 17.0$ years.

\section{DISCUSSION}

The aim of this study was to investigate the effect of BCG vaccination on the time of onset of T1D. We found that there is a difference related to the age of diagnosis in favor of BCG vaccinated patients. Previously published studies indicate possible mechanisms to explain the vaccine's effect on glucose metabolism and the function of pancreatic islets. 
In the autoimmune environment, there is an imbalance between Tregs and CTLs. This imbalance leads to increased destruction of pancreatic islet cells, giving a smaller number of Tregs that stabilize the immune response. Several studies have shown that not only BCG vaccination but also tuberculosis infection restore this imbalance of autoimmunity via the production of TNF. TNF activates Tregs and reduces the number of CTLs. This activation occurs via epigenetic changes in the signature genes of the Tregs. Results from trials in diabetic mice have indicated the efficacy of BCG vaccine on the regeneration of the pancreatic islet cells. On the contrary, residual $\mathrm{C}$ peptide levels in humans with a long duration of T1D after vaccination were undetectable. In case the vaccination occurs before the onset of diabetes, islets of the pancreas may be protected from autoimmunity. In our study, BCG vaccination was performed approximately at the age of 9 years. According to the aforementioned proposed mechanism, BCG vaccination may presented a protective effect in pancreatic cells from CTLs and autoimmunity. As a result, vaccinated subjects experiences a delay in developing T1D compared to the unvaccinated [4-11].

The hygiene hypothesis contends that in modern societies, there is a rise of autoimmune diseases because of the reduced exposure of the immune system to pathogens. In general, microorganisms shift host cells' metabolism to aerobic glycolysis. Recent data have indicated the relationship between glucose levels and immunometabolism. In patients with diabetes, immune cells like lymphocytes and macrophages are in an overactive state of oxidative phosphorylation for energy production. Oxidative phosphorylation uses Krebs cycle, producing ketones, and consequently, there is minimal need for glucose uptake. As a result, a greater amount of glucose remains extracellularly. In contrast, after BCG vaccination in patients with diabetes, aerobic glycolysis becomes the predominant metabolic pathway for energy production. Pentose phosphate shunt is activated, and membrane transportation of glucose is also accelerated. Glucose becomes the main fuel of immunometabolism, and fewer metabolites are funneled through the Krebs cycle. Trials have shown that purines which are metabolic products of pentose phosphate shunt are augmented, and as a result, levels of serum glucose tend to be lower. BCG vaccination regulates the imbalance between oxidative phosphorylation and aerobic glycolysis in lymphocytes. Our data suggest that this physiologic mechanism not only delays the onset of diabetes but also ameliorates serum glucose and $\mathrm{HbA1c}$ in diabetic patients [12-14].

Several studies in diabetic mice and humans suggest that not one but multiple BCG doses are necessary to prevent T1D. In a randomized, 8 -year, prospective trial, two doses of BCG vaccine were performed. After 3 years of clinical observation, HbA1c values had been reduced to near-normal levels and maintained for the next 5 years. mRNA sequencing and metabolomics demonstrated that a shift had occurred from oxidative phosphorylation to aerobic glycolysis during the trial. In our observational study, only a single dose of BCG vaccine had been performed at the age of 9 years old. Nevertheless, we have noticed a significant increase in the age of T1D diagnosis $[9,15]$.

\section{Limitations}

The main limitation of our study is the small sample size. Therefore, larger, high-quality trials with long follow-up periods are required to verify our findings.

\section{CONCLUSION}

The current trial showed that BCG vaccination, with a single dose at the age of 9 years old, probably delays T1D onset. Additional studies of children receiving multiple doses of BCG should be conducted to possibly prove prolongation of the disease-free interval.

\section{ACKNOWLEDGEMENTS}

We would like to express our special thanks of gratitude to the board of the Pan-Hellenic 
Federation of People with Diabetes, for their continuous help and support throughout the study.

Funding. No funding or sponsorship was received for this study. No funding or sponsorship was received for medical writing services for this manuscript.

Authorship. All named authors meet the International Committee of Medical Journal Editors (ICMJE) criteria for authorship for this article, take responsibility for the integrity of the work as a whole, and have given their approval for this version to be published.

Authors' Contributions. John Doupis developed the concept and the design of the study also having the overall supervision of the project. Konstantinos Kolokathis conducted the literature review and wrote the manuscript. Markopoulou Eftychia, Festas George, Papandreopoulou Vasiliki, Kallinikou Chrysoula, Antikidou Despina, Gemistou Golfo and Angelopoulos Theodoros contributed equally in data collection and management and Efthymiou Vasiliki performed the statistical analysis of the data.

Prior Presentation. The results of this study were partly disseminated at the 55th European Diabetes Epidemiology Group EDEG Annual Meeting as a virtual conference from 26 to 27 th of April 2021.

Disclosures. John Doupis, Konstantinos Kolokathis, Eftychia Markopoulou, Vasiliki Efthymiou, George Festas, Vasiliki Papandreopoulou, Chrysoula Kallinikou, Despina Antikidou, Golfo Gemistou and Theodoros Angelopoulos have nothing to disclose.

Compliance with Ethics Guidelines. The study protocol was conducted according to the Declaration of Helsinki and approved by the ethics committee of the Salamis Naval and Veterans Hospital, Salamis, Greece.

Data Availability. Participating patients with diagnosed T1D diabetes, provided information regarding age, gender, time of diagnosis and BCG vaccination status.

Open Access. This article is licensed under a Creative Commons Attribution-NonCommercial 4.0 International License, which permits any non-commercial use, sharing, adaptation, distribution and reproduction in any medium or format, as long as you give appropriate credit to the original author(s) and the source, provide a link to the Creative Commons licence, and indicate if changes were made. The images or other third party material in this article are included in the article's Creative Commons licence, unless indicated otherwise in a credit line to the material. If material is not included in the article's Creative Commons licence and your intended use is not permitted by statutory regulation or exceeds the permitted use, you will need to obtain permission directly from the copyright holder. To view a copy of this licence, visit http://creativecommons.org/licenses/by$\mathrm{nc} / 4.0 /$.

\section{REFERENCES}

1. IDF. Worldwide toll of diabetes. https://www. diabetesatlas.org/en/sections/worldwide-toll-ofdiabetes.html. Accessed Apr 21, 2021.

2. Arts RJW, Carvalho A, La Rocca C, et al. Immunometabolic pathways in BCG-induced trained immunity. Cell Rep. 2016;17(10):2562-71. https:// doi.org/10.1016/j.celrep.2016.11.011.

3. Ryu S, Kodama S, Ryu K, Schoenfeld DA, Faustman DL. Reversal of established autoimmune diabetes by restoration of endogenous beta cell function. J Clin Invest. 2001;108(1):63-72.

4. Okubo Y, Mera T, Wang L, Faustman DL. Homogeneous expansion of human T-regulatory cells via tumor necrosis factor receptor 2. Sci Rep. 2013;3(1): $1-11$.

5. Okubo Y, Torrey H, Butterworth J, et al. Treg activation defect in type 1 diabetes: correction with TNFR2 agonism Clin Transl Immunol. 2016;5(1): e56. https://doi.org/10.1038/cti.2015.43.

6. Faustman DL. The value of BCG and TNF in autoimmunity. 2nd ed. New York: Elsevier; 2018. p. 200. 
7. Liu Y, Wang L, Han R, et al. Two histone/protein acetyltransferases, CBP and p300, are indispensable for Foxp ${ }^{3+}$ T-regulatory cell development and function. Mol Cell Biol. 2014;34(21):15.

8. Kühtreiber WM, Faustman DL. BCG therapy for type 1 diabetes: restoration of balanced immunity and metabolism. Trends Endocrinol Metab. 2019;30(2):80-92.

9. Kühtreiber WM. Long-term reduction in hyperglycemia in advanced type 1 diabetes: the value of induced aerobic glycolysis with BCG vaccinations. NPJ Vaccines. 2018. https://doi.org/10.1038/ s41541-018-0062-8.

10. Kodama S, Kühtreiber W, Fujimura S, Dale EA, Faustman DL. Islet regeneration during the reversal of autoimmune diabetes in NOD mice. Science. 2003;302(5648):1223-7.

11. Ban L, Zhang J, Wang L, Kuhtreiber W, Burger D, Faustman DL. Selective death of autoreactive T cells in human diabetes by TNF or TNF receptor 2 agonism. Proc Natl Acad Sci. 2008;105(36):13644-9.

12. Faustman DL, Wang L, Okubo Y, et al. Proof-ofconcept, randomized, controlled clinical trial of Bacillus-Calmette-Guerin for treatment of longterm type 1 diabetes. PLoS One. 2012;7(8):16.

13. Cheng S-C, Quintin J, Cramer RA, et al. mTOR- and HIF-1 $\alpha$-mediated aerobic glycolysis as metabolic basis for trained immunity. Science. 2014;345(6204):1250684.

14. Bai G, Knapp GS, McDonough KA. Cyclic AMP signalling in mycobacteria: redirecting the conversation with a common currency. Cell Microbiol. 2010;13(3):349-58.

15. Shehadeh N, Etzioni A, Cahana A, et al. Repeated BCG vaccination is more effective than a single dose in preventing diabetes in non-obese diabetic (NOD) mice. Isr J Med Sci. 1997;33(11):711-5. 